\title{
Effect modification of perinatal exposure to air pollution and childhood asthma incidence
}

\author{
Éric Lavigne ${ }^{1,2}$, Marc-André Bélair ${ }^{3}$, Daniel Rodriguez Duque ${ }^{3}$, Minh T. Do ${ }^{4}$, \\ David M. Stieb ${ }^{2,5}$, Perry Hystad ${ }^{6}$, Aaron van Donkelaar ${ }^{7}$, Randall V. Martin ${ }^{7}$, \\ Daniel L. Crouse ${ }^{8}$, Eric Crighton ${ }^{3,9}$, Hong Chen ${ }^{10,11,12}$, Richard T. Burnett ${ }^{13}$, \\ Scott Weichenthal ${ }^{1,14}$, Paul J. Villeneuve ${ }^{15}$, Teresa To ${ }^{11,12,16}$, \\ Jeffrey R. Brook ${ }^{11,17}$, Markey Johnson ${ }^{1}$, Sabit Cakmak ${ }^{14}$, \\ Abdool S. Yasseen III 18,19,20 and Mark Walker ${ }^{18,19,20,21}$
}

@ERSpublications

Maternal asthma enhances the effect of air pollution during pregnancy on the risk of developing asthma in children http://ow.ly/eeWp30hSsIb

Cite this article as: Lavigne É, Bélair M-A, Rodriguez Duque D, et al. Effect modification of perinatal exposure to air pollution and childhood asthma incidence. Eur Respir J 2018; 51: 1701884 [https://doi.org/ 10.1183/13993003.01884-2017].

ABSTRACT Perinatal exposure to ambient air pollution has been associated with childhood asthma incidence; however, less is known regarding the potential effect modifiers in this association. We examined whether maternal and infant characteristics modified the association between perinatal exposure to air pollution and development of childhood asthma.

761172 births occurring between 2006 and 2012 were identified in the province of Ontario, Canada. Associations between exposure to ambient air pollutants and childhood asthma incidence (up to age 6 years) were estimated using Cox regression models.

110981 children with asthma were identified. In models adjusted for postnatal exposures, secondtrimester exposures to particulate matter with a $50 \%$ cut-off aerodynamic diameter $\leqslant 2.5 \mu \mathrm{m}$ (hazard ratio (HR) per interquartile range (IQR) increase 1.07, 95\% CI 1.06-1.09) and nitrogen dioxide (HR per IQR increase 1.06, 95\% CI 1.03-1.08) were associated with childhood asthma development. Enhanced impacts were found among children born to mothers with asthma, who smoked during pregnancy or lived in urban areas during pregnancy, males and children born preterm or of low birthweight.

Prenatal exposure to air pollution may have a differential impact on the risk of asthma development, according to maternal and infant characteristics.

\footnotetext{
This article has supplementary material available from erj.ersjournals.com

Received: June 262017 | Accepted after revision: Jan 142018

Support statement: This study was funded by the Public Health Agency of Canada and by the Clean Air Regulatory Agenda programme of Health Canada. This study was supported by the Institute for Clinical Evaluative Sciences (ICES), which is funded by an annual grant from the Ontario Ministry of Health and Long-Term Care. The opinions, results and conclusions reported in this paper are those of the authors and are independent from the funding sources. Funding information for this article has been deposited with the Crossref Funder Registry.

Conflict of interest: None declared.
}

The content of this work is copyright of the authors or their employers. Design and branding are copyright @ERS 2018. This version is distributed under the terms of the Creative Commons Attribution Non-Commercial Licence 4.0. 
Affiliations: ${ }^{1}$ Air Health Science Division, Health Canada, Ottawa, ON, Canada. ${ }^{2}$ School of Epidemiology and Public Health, University of Ottawa, Ottawa, ON, Canada. ${ }^{3}$ Institute for Clinical Evaluative Sciences, Ottawa, ON, Canada. ${ }^{4}$ Surveillance and Epidemiology Division, Public Health Agency of Canada, Ottawa, ON, Canada. ${ }^{5}$ Population Studies Division, Health Canada, Vancouver, BC, Canada. ${ }^{6}$ College of Public Health and Human Sciences, Oregon State University, Corvallis, OR, USA. ${ }^{7}$ Dept of Physics and Atmospheric Science, Dalhousie University, Halifax, NS, Canada. ${ }^{8}$ Dept of Sociology, University of New Brunswick, Fredericton, NB, Canada. ${ }^{9}$ Dept of Geography, Environment and Geomatics, University of Ottawa, Ottawa, ON, Canada. ${ }^{10}$ Public Health Ontario, Toronto, ON, Canada. ${ }^{11}$ Dalla Lana School of Public Health, University of Toronto, Toronto, ON, Canada. ${ }^{12}$ Institute for Clinical Evaluative Sciences, Toronto, ON, Canada. ${ }^{13}$ Population Studies Division, Health Canada, Ottawa, ON, Canada. ${ }^{14}$ Dept of Epidemiology, Biostatistics and Occupational Health, McGill University, Montreal, QC, Canada. ${ }^{15}$ Dept of Health Sciences, Carleton University, Ottawa, ON, Canada. ${ }^{16} \mathrm{Child}$ Health Evaluative Sciences, The Hospital for Sick Children, Toronto, ON, Canada. ${ }^{17}$ Air Quality Research Division, Environment Canada, Downsview, ON, Canada. ${ }^{18}$ Ottawa Hospital Research Institute, Ottawa, ON, Canada. ${ }^{19}$ Better Outcomes Registry and Network Ontario, Ottawa, ON, Canada. ${ }^{20}$ Children's Hospital of Eastern Ontario Research Institute, Ottawa, ON, Canada. ${ }^{21}$ Dept of Obstetrics and Gynecology, University of Ottawa, Ottawa, ON, Canada.

Correspondence: Éric Lavigne, Air Health Science Division, Health Canada, 269 Laurier Avenue West, Mail Stop 4903B, Ottawa, ON, Canada, K1A 0K9. E-mail: eric.lavignelacanada.ca

\section{Introduction}

Asthma is one of the leading prevalent paediatric chronic diseases in the world [1]. Evidence shows that both genetic and environmental factors are responsible for the development of asthma [2]. A number of epidemiological studies have reported associations between ambient air pollution and childhood asthma incidence $[3,4]$ as well as lung function deficits [5-8], with some studies suggesting that this relationship may begin in utero [5, 9-16]. However, most studies have been restricted to urban populations living in the vicinity of air pollution monitoring stations and therefore, further evidence is required when comparing urban and rural populations in childhood asthma risk. In addition, further evidence is needed in order to disentangle the importance of different prenatal and postnatal periods of exposure for childhood asthma incidence.

One of the strongest risk factors for childhood asthma development is the presence of a maternal history of asthma [17-19]. Asthma is the most common chronic disease to affect pregnant women [20], but no studies to date have evaluated whether the effect of prenatal exposure to outdoor air pollution on childhood asthma incidence is enhanced among children of mothers with a history of asthma, or whether their joint effects increase risk of childhood asthma. Sex differences in the relationship between exposure to outdoor air pollution and risk of childhood asthma development have been observed, but inconsistent findings have been reported $[9,11,12]$. For instance, it is hypothesised that male infants could be more susceptible to the harmful effects of air pollution due to their specific pulmonary phenotype compared to female infants [7]. One previous study showed that infants born preterm and of low birthweight are at increased risk of developing asthma when exposed to increased levels of outdoor air pollution [11], but this requires further investigation. Other potential modifying factors for which further evidence is needed include maternal smoking during pregnancy and maternal atopy [21].

In this study, we made use of a large population-based sample encompassing both urban and rural areas to evaluate the associations between exposures during pregnancy and early postnatal life to nitrogen dioxide $\left(\mathrm{NO}_{2}\right)$ as a marker of traffic-related air pollution and particulate matter with a $50 \%$ cut-off aerodynamic diameter $\leqslant 2.5 \mu \mathrm{m}$ (PM2.5) as a marker of the complex mixture of outdoor air pollution, with childhood asthma incidence. We further examined whether these associations were modified by maternal history of asthma, maternal atopy, maternal smoking during pregnancy, infant sex, gestational age, birthweight and maternal place of residence (urban/rural areas).

\section{Methods}

\section{Study population and design}

We identified a retrospective cohort of pregnant women who gave birth to live-born singleton infants in the province of Ontario, Canada. Mother-infant-pair data were obtained from a province-wide birth registry for the time period between April 1, 2006 and March 31, 2012 [22]. Each mother's residential location(s) during pregnancy was obtained based on residential postal code(s) reported from health administrative databases. Gestational age was determined by first trimester ultrasound dating and the mother's last menstrual period (see the online supplementary material for more details on study population).

\section{Ascertainment of childhood asthma}

We identified incident childhood asthma cases (International Classification of Diseases, 10th revision (ICD-10): J45) for the time period April 1, 2006 to March 31, 2013 using the Ontario Asthma Surveillance 
Information System, which is a population-based system that identifies and tracks individuals living with asthma in Ontario, Canada [23]. A previously validated case definition of asthma was used to identify individuals with asthma (online supplementary material). We examined incident childhood asthma diagnosed between birth and $<6$ years of age, consistent with prior literature $[9,11]$. The data linkage process across databases and linkage with exposure estimates is depicted in online supplementary figure S1. For instance, 222864 participants could be assigned exposure estimates to both $\mathrm{PM} 2.5$ and $\mathrm{NO}_{2}$ (exposure assessment described below). These datasets were linked using unique encoded identifiers and analysed at the Institute for Clinical Evaluative Sciences.

\section{Exposure assessment}

Air pollution exposure estimates were assigned to the geographical coordinates representing the centroid of each subject's residential 6-digit postal code, as previously described [24, 25]. In brief, we assigned exposure to PM2.5 during each trimester of pregnancy and during the first year of life, and for the cumulative exposure after birth, based on monthly satellite surfaces at a $1 \times 1 \mathrm{~km}$ resolution [26, 27]. Estimates were obtained for trimester-specific periods of exposure and were averaged to obtain estimates for the entire pregnancy.

A national land use regression (LUR) model was used to assign prenatal and postnatal exposure estimates to ambient $\mathrm{NO}_{2}$ to residential postal codes $[25,28,29]$. We applied a temporal adjustment to the LUR $\mathrm{NO}_{2}$ model, which allowed us to more precisely obtain estimates of exposure to $\mathrm{NO}_{2}$ during each trimester of pregnancy, during the first year of life and for the cumulative exposure after birth. We captured residential mobility during pregnancy by calculating the time spent at each postal code and assigned pollutant exposure accordingly. Additional details regarding exposure assessment methods to ambient air pollutants can be found in the online supplementary material.

\section{Covariates}

The following variables were available from health administrative databases and were investigated as potential confounders and/or effect modifiers [9, 11]: maternal age at delivery $(<20$ years, $20-34$ years, $\geqslant 35$ years or missing data), infant sex, parity $(0,1$ or $\geqslant 2)$, maternal intention to breastfeed on discharge (yes, no or missing data), maternal cigarette smoking anytime during pregnancy (yes, no or missing data), maternal history of asthma [30], maternal atopy status (see supplemental material regarding maternal asthma and atopy case definitions) [9], gestational age (in weeks), birthweight and an indicator for urban/ rural place of residence (see online supplementary material regarding details on urban/rural indicator). Exposure to residential greenness during pregnancy was obtained using the satellite-derived normalised difference vegetation index (NDVI) and exposure was assigned at the postal code level [31] (see online supplementary material regarding exposure assessment of NDVI). In addition, we abstracted three contextual socioeconomic status (SES) variables (i.e. median family income, proportion of visible minority and percentage of females aged 25-64 years who completed post-secondary education).

\section{Statistical analysis}

We employed random-effects Cox proportional hazards models to evaluate the associations between exposure to air pollution during pregnancy and incidence of childhood asthma, in which random effects were represented by two levels of spatial clusters: census division (equivalent in size to a county) and census tract within census divisions [24,32]. Follow-up time (i.e. child age in days) was measured from birth until incidence of childhood asthma, death, ineligibility for provincial health insurance or end of follow-up. We conducted analyses for the whole pregnancy, trimester-specific exposures, first year of life and childhood exposures. Models were conducted with and without mutual adjustment for average pregnancy exposure, first year of life exposure and cumulative exposure after birth. For instance, in the mutually adjusted models, effect estimates for the average pregnancy exposure were additionally adjusted for the first year of life exposure and the cumulative exposure after birth. Hazard ratios for trimester-specific exposures in mutually adjusted models accounted for average pregnancy exposure, first year of life exposure and cumulative exposure after birth. Additionally, we adjusted for pregnancy average exposure in postnatal exposure models. Proportional hazards assumptions were verified by adding the cross product of each variable with the natural logarithm of the time variable, but we did not find any significant violations of this assumption $(\mathrm{p}>0.05)$. Results are expressed as the hazard ratio (HR) and $95 \%$ confidence interval corresponding to an increase across the interquartile range (IQR) of $\mathrm{NO}_{2}$ and $\mathrm{PM} 2.5$. Concentration response curves were evaluated using recently published methods [33].

We conducted stratified analyses to assess potential effect modification by selected individual (i.e. maternal history of asthma, maternal atopy status, maternal smoking during pregnancy, birthweight, gestational age and infant sex) and contextual (i.e. urban/rural status) characteristics. We evaluated the significance of effect modification on the multiplicative scale by including a cross product interaction term between each 
exposure of interest (i.e. PM2.5 and $\mathrm{NO}_{2}$ ) and each characteristic. Wald's method was used to assess the presence of interaction on the multiplicative scale. Effect modification was considered statistically significant if the interaction term $\mathrm{p}$-value was $<0.05$. In addition, we investigated the interaction between air pollution and maternal asthma as well as maternal atopy on the additive scale using the relative excess risk due to interaction (RERI), given that joint effects are also of interest. We categorised PM2.5 and $\mathrm{NO}_{2}$ in quartiles for this analysis. We used mothers without asthma (or mothers without an atopic disease) in the first exposure quartiles of either PM2.5 or $\mathrm{NO}_{2}$ as the reference category for the following groups (annotations in parentheses): presence of maternal asthma (or maternal atopy) and fourth quartile exposure of either $\mathrm{PM} 2.5$ or $\mathrm{NO}_{2}\left(\mathrm{HR}_{10}\right)$; absence of maternal asthma (or maternal atopy) and first quartile exposure of either $\mathrm{PM} 2.5$ or $\mathrm{NO}_{2}\left(\mathrm{HR}_{01}\right)$; presence of maternal asthma (or maternal atopy) and fourth quartile exposure of either $\mathrm{PM} 2.5$ or $\mathrm{NO}_{2}\left(\mathrm{HR}_{11}\right)$. We calculated the RERI as $\mathrm{HR}_{11}-\mathrm{HR}_{10}-\mathrm{HR}_{01}+1$. A RERI of 0 indicates no interaction and a statistically significant RERI $>0$ indicates the presence of supra-additivity or synergistic interaction [34]. The RERI and its 95\% confidence interval were calculated according to the $\delta$-method [35].

We conducted a number of sensitivity analyses including stratifying analyses by the child's age at diagnosis, restricting our analysis according to subjects that did not move during pregnancy and conducting two-pollutant models. Statistical analyses were peformed in R (version 3.0.1; www.r-project.org), using the "coxme" package. Ethics approval for this study was granted by the research ethics boards of Health Canada, the Children's Hospital of Eastern Ontario and the Ottawa Health Science Network.

\section{Results}

\section{Descriptive statistics}

761172 singleton live births occurring between April 1, 2006 and March 31, 2012 were identified in the province of Ontario, Canada (table 1). Among these, 110981 children developed asthma before the age of 6 years with a mean age at asthma diagnosis of 2.1 years. Children with asthma had a smaller birthweight $(3327.9 \pm 596.3 \mathrm{~g}$ versus $3397.1 \pm 540.5 \mathrm{~g})$, a slightly shorter gestational age $(38.6 \pm 2.1$ weeks versus $38.9 \pm 1.7$ weeks) and were more frequently born to mothers with a history of asthma ( $8.4 \%$ versus $5.6 \%$ ). There was a male predominance among asthmatic children (60.5\% versus $49.8 \%$ ). Furthermore, 45443 mothers were found to have a history of asthma.

The IQR for PM2.5 was $3.7 \mu \mathrm{g} \cdot \mathrm{m}^{-3}$ and the IQR for $\mathrm{NO}_{2}$ was $8.6 \mathrm{ppb}$ over the period of the whole pregnancy (table 2). PM2.5 was moderately correlated with $\mathrm{NO}_{2}$ during the entire pregnancy period $(\mathrm{r}=0.49)$ (online supplementary table S1). Moderate correlations were observed between trimester-specific periods and exposures after birth to PM2.5 ( $\mathrm{r}=0.54-0.74)$. In addition, moderate Pearson correlations were found between specific periods of exposure to $\mathrm{NO}_{2}(\mathrm{r}=0.51-0.78)$. Average long-term air pollution exposure (i.e. combining pregnancy exposure and exposures after birth) in mothers with asthma was slightly lower $\left(7.2 \mu \mathrm{g} \cdot \mathrm{m}^{-3}\right.$ for $\mathrm{PM} 2.5$ and $12.5 \mathrm{ppb}$ for $\left.\mathrm{NO}_{2}\right)$ compared to mothers without asthma $\left(7.4 \mu \mathrm{g} \cdot \mathrm{m}^{-3}\right.$ for $\mathrm{PM} 2.5$ and $13.3 \mathrm{ppb}$ for $\mathrm{NO}_{2}$ ) (results not shown in tables). We observed negative correlations between long term PM2.5 $(r=-0.44)$ and $\mathrm{NO}_{2}(\mathrm{r}=-0.43)$ exposures with residential greenness exposure during pregnancy.

\section{Air pollution and childhood asthma associations}

The associations between exposure to $\mathrm{PM} 2.5$ and $\mathrm{NO}_{2}$ on childhood asthma incidence over specific time periods of pregnancy are presented in table 2. We found that additional adjustment for the pregnancy average exposure and the exposures after birth decreased effect estimates in most associations. In fact, we found statistically significant hazard ratios for exposures to $\mathrm{NO}_{2}(1.06,95 \%$ CI 1.03-1.08) and PM2.5 (1.07, 95\% CI 1.06-1.09) only for the second trimester when additionally adjusting for exposures to the selected pollutant during the whole pregnancy and exposures after birth. Supplementary analyses using concentration-response functions using natural cubic splines with three degrees of freedom [33] for $\mathrm{NO}_{2}$ and PM2.5 during the second trimester with adjustment for all the same covariates reported in table 2 (i.e. mutually adjusted model) confirmed linearity of associations with incident asthma throughout the distribution of air pollutant concentrations (online supplementary figure S2). Exposure to residential greenness during pregnancy appeared to be independently associated with reduced risk of childhood asthma (HR 0.83, 95\% CI 0.82-0.83) after adjustment for air pollution measures (result not shown).

\section{Air pollution and childhood asthma effect modification}

Stratified analyses, adjusted for a number of covariates including the mutual adjustment for pregnancy average and exposures after birth, are presented in tables 3 and 4. Analyses stratified according to gestational age showed that children born preterm were at highest risk (HR 1.11,95\% CI 1.08-1.14) of developing childhood asthma per $9.7 \mathrm{ppb}$ increase in exposure to $\mathrm{NO}_{2}$ during the second trimester when compared to the risk for those born at term (HR 1.06, 95\% CI 1.03-1.08) (p-value=0.04) (table 3). 
TABLE 1 Demographic and socioeconomic characteristics of study participants

\begin{tabular}{|c|c|c|c|}
\hline & Total cohort & Asthmatic children & Nonasthmatic children \\
\hline Maternal age years & $30.0 \pm 5.5$ & $30.1 \pm 5.5$ & $30.0 \pm 5.5$ \\
\hline Birthweight $\mathrm{g}$ & $3387.0 \pm 549.6$ & $3327.9 \pm 596.3$ & $3397.9 \pm 540.6$ \\
\hline \multicolumn{4}{|l|}{ Infant sex } \\
\hline Male & 390665 (51.3) & $67171(60.5)$ & 323484 (49.8) \\
\hline 0 & $385336(50.6)$ & 55777 (50.3) & 329559 (50.7) \\
\hline 1 & $259543(34.1)$ & $39004(35.1)$ & 220539 (33.9) \\
\hline$\geqslant 2$ & 116293 (15.3) & $16200(14.6)$ & $100093(15.4)$ \\
\hline \multicolumn{4}{|l|}{ Intention to breastfeed } \\
\hline Yes & $606236(79.6)$ & 87503 (78.8) & 518733 (79.8) \\
\hline No & 64787 (8.5) & 9607 (8.7) & 55180 (8.5) \\
\hline \multicolumn{4}{|l|}{ Maternal asthma } \\
\hline Yes & $45443(6.0)$ & $9299(8.4)$ & $36144(5.6)$ \\
\hline No & $715729(94.0)$ & $101682(91.6)$ & $614047(94.4)$ \\
\hline \multicolumn{4}{|l|}{ Median family income } \\
\hline Quintile 1 & 149838 (19.7) & 23566 (21.2) & $126272(19.4)$ \\
\hline Quintile 2 & 149405 (19.6) & 21798 (19.6) & $127607(19.6)$ \\
\hline Quintile 3 & 150339 (19.8) & $20974(18.9)$ & 129365 (19.9) \\
\hline Quintile 4 & $149788(19.7)$ & 21826 (19.7) & $127962(19.7)$ \\
\hline Quintile 5 & $149872(19.7)$ & 21115 (19.0) & $128757(19.8)$ \\
\hline Missing & $11930(1.6)$ & 1702 (1.5) & $10228(1.6)$ \\
\hline \multicolumn{4}{|c|}{ Percentage of females who completed post-secondary education (age $\geqslant 25$ years) } \\
\hline Quintile 1 & $124580(19.7)$ & $16649(18.0)$ & 107931 (20.0) \\
\hline Quintile 3 & $149768(19.7)$ & $21185(19.1)$ & $128583(19.8)$ \\
\hline Quintile 4 & $149744(19.7)$ & $24620(22.2)$ & $125124(19.2)$ \\
\hline Quintile 5 & 149777 (19.7) & $28527(25.7)$ & $121250(18.6)$ \\
\hline Missing & $12343(1.6)$ & $1759(1.6)$ & $10584(1.6)$ \\
\hline
\end{tabular}

Data are presented as $n$, mean \pm SD or $n(\%)$.

Stratification by maternal place of residence showed that children born to mothers who were living in urban areas during their pregnancy had a heightened impact of exposure to $\mathrm{NO}_{2}$ during the first trimester on childhood asthma development ( $p$-value for effect modification $=0.04$ ). In addition, we found statistically significant effect modification by infant sex, with males having higher risks of developing asthma, for exposures to PM2.5 in trimesters 1 and 2 (p-values for effect modification $\leqslant 0.04$ ) (table 4). In addition, stratified regressions revealed that low-birthweight infants, those born preterm and those born to mothers who smoked during pregnancy were at an increased risk for asthma when exposed to increased levels of PM2.5 compared to their counterparts (p-values for effect modification $\leqslant 0.04$ ). Given that maternal smoking during pregnancy is negatively associated with gestational age and birthweight, we restricted our analysis of effect modification by gestational age and birthweight to those who did not smoke during pregnancy. Results for effect modification by gestational age and birthweight remained statistically significant ( $\mathrm{p}$-values for effect modification $\leqslant 0.04$; results not shown).

The risk of childhood asthma diagnosed before 6 years of age was significantly increased when evaluating effect modification on the additive scale for the combined effect of maternal asthma and exposure to $\mathrm{NO}_{2}$ 
TABLE 2 Hazard ratios (HR) and $95 \%$ confidence intervals for the associations between nitrogen dioxide $\left(\mathrm{NO}_{2}\right)($ per interquartile range (IQR)) and particulate matter with a $50 \%$ cut-off aerodynamic diameter $\leqslant 2.5 \mu \mathrm{m}$ (PM2.5) (per IQR) over specific periods and childhood asthma risk

\begin{tabular}{|c|c|c|c|c|c|c|c|c|}
\hline & \multicolumn{4}{|c|}{$\mathrm{NO}_{2}$} & \multicolumn{4}{|c|}{ PM2.5 } \\
\hline & $\begin{array}{l}\text { Obstetric } \\
\text { cases }\end{array}$ & $\begin{array}{l}\text { IQR } \\
\text { ppb }\end{array}$ & Adjusted model ${ }^{\#}$ & $\begin{array}{c}\text { Mutually } \\
\text { adjusted model }\end{array}$ & $\begin{array}{c}\text { Obstetric } \\
\text { cases }\end{array}$ & $\begin{array}{c}\text { IQR } \\
\mu \mathrm{g} \cdot \mathrm{m}^{-3}\end{array}$ & Adjusted model ${ }^{\#}$ & $\begin{array}{c}\text { Mutually } \\
\text { adjusted model" }\end{array}$ \\
\hline 2nd trimester & 27874 & 9.7 & $1.19(1.17-1.21)$ & 1.06 (1.03-1.08) & 84398 & 3.9 & $1.09(1.08-1.10)$ & $1.07(1.06-1.09)$ \\
\hline 3rd trimester & 27260 & 9.5 & $0.99(0.97-1.01)$ & $0.98(0.96-1.00)$ & 84056 & 3.8 & $1.02(1.00-1.04)$ & $1.01(0.99-1.03)$ \\
\hline Entire pregnancy & 27213 & 8.6 & $1.09(1.07-1.12)$ & $1.02(0.99-1.05)$ & 83470 & 3.7 & $1.02(0.99-1.04)$ & $1.01(0.99-1.04)$ \\
\hline
\end{tabular}

Data are presented as $\mathrm{n}$ or HR $(95 \% \mathrm{Cl}) .{ }^{*}$ : model adjusted for maternal age at delivery, infant sex, parity, breastfeeding status at the time of discharge, maternal smoking during pregnancy, maternal atopy, gestational age, birthweight, residential greenness exposure during pregnancy, dissemination area median family income, dissemination area proportion of population who are visible minority and dissemination area proportion of the adult female population aged 25-64 years old who completed post-secondary education; " includes all variables in the adjusted model plus the average pregnancy exposure to the selected pollutant, the first year of life exposure to the selected pollutant and cumulative exposure after birth to the selected pollutant IQR.

(table 5). The highest effect was observed among children whose exposure to $\mathrm{NO}_{2}$ during the second trimester was in the highest category of exposure (i.e. 4th quartile) and whose mothers had asthma (HR 1.87, 95\% CI 1.69-2.07). RERI estimates for joint effects of high $\mathrm{NO}_{2}$ exposure and maternal asthma on childhood asthma incidence exceeded 0 for second trimester exposure and RERI was statistically significant, which suggests supra-additivity (i.e. synergistic effects) for interaction on the additive scale. No evidence of effect modification on the additive scale was found for the other associations investigated (tables 5 and 6) and for effect modification by maternal atopy on the additive scale (results not shown).

\section{Sensitivity analyses}

We conducted a number of sensitivity analyses. When stratifying the analysis by the child's age at diagnosis ( $<1$ year versus $1-5$ years of age), we found no differences in effect estimates (data not shown). In addition, we found $<3 \%$ differences in risk estimates when comparing those that did not move during pregnancy or during childhood years compared to all subjects (results not shown). In two-pollutant models, we found that effect estimates for both pollutants decreased slightly when adjusting for the other pollutant (online supplementary table S2). In addition, we found that effect estimates for single-pollutant models restricted to those where both exposure estimates could be assigned were similar to the overall models (online supplementary table S2). The same pattern was observed when investigating two-pollutant models when evaluating effect modification (i.e. $<3 \%$ difference in risk estimates; results not shown). Results of the evaluation of the joint effects between $\mathrm{NO}_{2}$ during the second trimester and maternal asthma were robust to adjustment for cumulative exposures after birth (results not shown). In addition, we investigated associations restricted to term births weighing $\geqslant 2500$ grams (i.e. without adjustment for birthweight and gestational age, since these factors may be on the causal pathway between prenatal exposure to air pollution and childhood asthma), but risk estimates were materially unchanged (results not shown). We investigated whether parity was an effect modifier in the relationship between air pollution variables and asthma development, but findings did not reveal presence of effect modification ( $p$-value for effect modification $\geqslant 0.11$; results not shown).

\section{Discussion}

In this population-based study in the largest province of Canada, we examined associations between prenatal and early postnatal life exposure to air pollution and early childhood asthma incidence. We found that second-trimester exposures to $\mathrm{NO}_{2}$ and $\mathrm{PM} 2.5$ were associated with increased risks of developing asthma in children up to 6 years of age. In addition, we found evidence suggestive that children of mothers who had asthma and who were in the upper quartile of exposure to $\mathrm{NO}_{2}$ during the second trimester were approximately two times more at risk of developing asthma before 6 years of age. Increased effects of exposure to air pollution on childhood asthma incidence were found for those born preterm or of low birthweight, males, those born to mothers who smoked during pregnancy and those born to mothers living in urban areas during pregnancy. 
TABLE 3 Adjusted ${ }^{\#}$ hazard ratios (HR) and $95 \%$ confidence intervals for the associations between nitrogen dioxide $\left(\mathrm{NO}_{2}\right)$ per interquartile range over specific periods and childhood asthma risk, stratified by selected characteristics

\begin{tabular}{|c|c|c|c|c|c|c|}
\hline & 1st trimester & 2nd trimester & 3rd trimester & $\begin{array}{c}\text { Entire } \\
\text { pregnancy }\end{array}$ & $\begin{array}{l}\text { First year of } \\
\text { life }\end{array}$ & $\begin{array}{c}\text { Childhood cumulative } \\
\text { exposure }\end{array}$ \\
\hline \multicolumn{7}{|l|}{ Maternal asthma } \\
\hline No & $1.02(0.99-1.04)$ & $1.06(1.03-1.08)$ & $0.98(0.96-1.00)$ & $1.02(0.99-1.05)$ & $1.03(0.99-1.06)$ & $1.00(0.97-1.03)$ \\
\hline $\begin{array}{c}p \text {-value for effect } \\
\text { modification }\end{array}$ & 0.65 & 0.38 & 0.42 & 0.49 & 0.64 & 0.75 \\
\hline \multicolumn{7}{|l|}{ Maternal atopy } \\
\hline No & $1.02(1.00-1.05)$ & $1.06(1.03-1.08)$ & $0.98(0.96-1.00)$ & $1.02(0.99-1.05)$ & $1.03(1.00-1.06)$ & $1.00(0.97-1.03)$ \\
\hline $\begin{array}{l}\text { p-value for effect } \\
\text { modification }\end{array}$ & 0.21 & 0.16 & 0.78 & 0.34 & 0.32 & 0.92 \\
\hline \multicolumn{7}{|c|}{ Maternal smoking during pregnancy } \\
\hline Yes & $1.08(1.03-1.13)$ & $1.11(1.05-1.16)$ & $1.05(1.00-1.10)$ & $1.07(1.01-1.13)$ & $1.05(1.00-1.10)$ & $1.00(0.93-1.07)$ \\
\hline No & $1.02(1.00-1.05)$ & $1.06(1.03-1.08)$ & $1.00(0.98-1.02)$ & $1.02(0.99-1.05)$ & $1.03(1.00-1.06)$ & $1.00(0.97-1.03)$ \\
\hline \multicolumn{7}{|l|}{ Infant sex } \\
\hline $\begin{array}{l}p \text {-value for effect } \\
\text { modification }\end{array}$ & 0.55 & 0.88 & 0.74 & 0.92 & 0.57 & 0.84 \\
\hline \multicolumn{7}{|c|}{ Maternal place of residence } \\
\hline Urban & $1.04(1.01-1.07)$ & $1.06(1.03-1.08)$ & $1.00(0.98-1.03)$ & $1.02(0.99-1.05)$ & $1.04(1.02-1.06)$ & $1.00(0.97-1.03)$ \\
\hline Rural & $0.75(0.55-1.01)$ & $0.98(0.71-1.34)$ & $1.00(0.67-1.45)$ & $0.99(0.58-1.55)$ & $1.00(0.60-1.59)$ & $0.99(0.61-1.65)$ \\
\hline $\begin{array}{c}p \text {-value for effect } \\
\text { modification }\end{array}$ & 0.04 & 0.51 & 0.97 & 0.88 & 0.75 & 0.88 \\
\hline \multicolumn{7}{|l|}{ Gestational age } \\
\hline$<37$ weeks & $1.02(1.00-1.05)$ & $1.11(1.08-1.14)$ & $1.02(0.98-1.06)$ & $1.05(1.00-1.09)$ & $1.04(0.99-1.09)$ & $1.00(0.95-1.05)$ \\
\hline$\geqslant 37$ weeks & $1.03(1.00-1.06)$ & $1.06(1.03-1.08)$ & $0.98(0.96-1.01)$ & $1.02(0.99-1.05)$ & $1.03(1.00-1.06)$ & $1.00(0.98-1.02)$ \\
\hline $\begin{array}{l}p \text {-value for effect } \\
\text { modification }\end{array}$ & 0.44 & 0.04 & 0.33 & 0.43 & 0.86 & 0.98 \\
\hline
\end{tabular}

Many studies have investigated the association between childhood exposure to air pollution and asthma onset in children [3,4], but few studies have investigated the effect of exposure to air pollution during specific periods of pregnancy on the risk of development of childhood asthma [9, 13, 14]. In a study conducted in China among 2598 children, exposure to increased levels of $\mathrm{NO}_{2}$ during the second trimester was associated with the development of asthma (odds ratio 1.72, 95\% CI 1.022.97) [13]. A study conducted in Boston (MA, USA) evaluated the effect of weekly exposures to $\mathrm{PM} 2.5$ during pregnancy on the development of asthma among 736 full-term children [9, 14]. They found that PM2.5 exposure during the second trimester was associated with asthma development by the age of 6 years, but only among males. Additionally, Morales et al. [5] showed that second-trimester $\mathrm{NO}_{2}$ exposure was associated with decreased lung function at 4.5 years of age. Therefore, findings of our study for an effect of exposure to $\mathrm{NO}_{2}$ and $\mathrm{PM} 2.5$ during the second trimester of pregnancy on childhood asthma incidence are somewhat consistent with prior literature. Exposure to air pollution in utero may potentially have harmful effects on critical periods of development of the immune and respiratory systems [36]. In particular, lung development in the second trimester of pregnancy may be 
TABLE 4 Adjusted ${ }^{\#}$ hazard ratios (HR) and 95\% confidence intervals for the associations between particulate matter with a $50 \%$ cut-off aerodynamic diameter $\leqslant 2.5 \mu \mathrm{m}$ (PM2.5) per interquartile range over specific periods and childhood asthma risk, stratified by selected characteristics

\begin{tabular}{|c|c|c|c|c|c|c|}
\hline & 1st trimester & 2nd trimester & 3rd trimester & $\begin{array}{c}\text { Entire } \\
\text { pregnancy }\end{array}$ & $\begin{array}{l}\text { First year of } \\
\text { life }\end{array}$ & $\begin{array}{c}\text { Childhood cumulative } \\
\text { exposure }\end{array}$ \\
\hline \multicolumn{7}{|l|}{ Maternal asthma } \\
\hline No & $1.01(1.00-1.03)$ & $1.07(1.06-1.09)$ & $1.01(0.99-1.03)$ & $1.01(0.98-1.04)$ & $0.99(0.98-1.00)$ & $1.00(0.99-1.01)$ \\
\hline $\begin{array}{c}p \text {-value for effect } \\
\text { modification }\end{array}$ & 0.26 & 0.92 & 0.67 & 0.81 & 0.88 & 0.94 \\
\hline No & $1.01(0.99-1.03)$ & $1.07(1.06-1.09)$ & $1.01(0.99-1.03)$ & $1.01(0.99-1.04)$ & $0.98(0.97-1.01)$ & $1.00(0.98-1.01)$ \\
\hline $\begin{array}{l}p \text {-value for effect } \\
\text { modification }\end{array}$ & 0.35 & 0.34 & 0.61 & 0.55 & 0.48 & 0.72 \\
\hline \multicolumn{7}{|c|}{ Maternal smoking during pregnancy } \\
\hline Yes & $1.07(1.03-1.12)$ & $1.12(1.09-1.14)$ & $1.05(1.02-1.08)$ & $1.07(1.04-1.11)$ & $1.00(0.98-1.03)$ & $0.99(0.96-1.01)$ \\
\hline No & $1.01(1.00-1.03)$ & $1.07(1.06-1.09)$ & $1.01(0.99-1.03)$ & $1.01(0.98-1.04)$ & $0.99(0.98-1.00)$ & $0.99(0.98-1.00)$ \\
\hline Female & $1.00(0.97-1.02)$ & $1.05(1.01-1.09)$ & $1.00(0.98-1.03)$ & $1.01(0.99-1.04)$ & $1.00(0.98-1.01)$ & $1.00(1.00-1.01)$ \\
\hline $\begin{array}{l}p \text {-value for effect } \\
\text { modification }\end{array}$ & 0.04 & 0.04 & 0.35 & 0.12 & 0.78 & 0.96 \\
\hline \multicolumn{7}{|c|}{ Maternal place of residence } \\
\hline Urban & $1.01(1.00-1.03)$ & $1.08(1.06-1.10)$ & $1.01(1.00-1.04)$ & $1.01(0.99-1.04)$ & $1.00(0.98-1.02)$ & $1.00(0.99-1.01)$ \\
\hline Rural & $1.02(0.98-1.07)$ & $1.06(1.00-1.11)$ & $1.00(0.95-1.06)$ & $1.01(0.98-1.04)$ & $1.00(0.95-1.07)$ & $1.00(0.95-1.06)$ \\
\hline $\begin{array}{l}p \text {-value for effect } \\
\text { modification }\end{array}$ & 0.42 & 0.36 & 0.60 & 0.86 & 0.67 & 0.76 \\
\hline \multicolumn{7}{|l|}{ Gestational age } \\
\hline$<37$ weeks & $1.06(1.03-1.10)$ & $1.13(1.09-1.17)$ & $1.03(0.99-1.07)$ & $1.04(1.00-1.09)$ & $1.00(0.95-1.05)$ & $1.00(0.95-1.06)$ \\
\hline$\geqslant 37$ weeks & $1.01(1.00-1.03)$ & $1.07(1.06-1.09)$ & $1.01(0.99-1.03)$ & $1.01(0.99-1.04)$ & $0.99(0.98-1.00)$ & $1.00(1.00-1.01)$ \\
\hline $\begin{array}{l}\text { p-value for effect } \\
\text { modification }\end{array}$ & 0.04 & 0.03 & 0.28 & 0.47 & 0.68 & 0.85 \\
\hline
\end{tabular}

affected through an increase in inflammation and airway hyperresponsiveness, which may enhance susceptibility to asthma $[9,37-39]$.

We found that exposure to air pollution during the first year of life with additional adjustment for pregnancy exposure was not associated with childhood asthma development. Although few studies have attempted to disentangle these relationships, Morales et al. [5] found that exposure to outdoor air pollutants in early postnatal life was not associated with lung function deficits at preschool age. However, a recent study showed that exposure to traffic-related air pollution in infancy is negatively associated with forced expiratory volume in $1 \mathrm{~s}$ at 16 years of age [8]. This could imply that effects of exposure during the first year of life may not be long enough to have an impact on childhood asthma development. Therefore, further studies are required to disentangle the impact of prenatal and postnatal exposure to air pollution on development of respiratory outcomes in later childhood and adolescence. 
TABLE 5 Adjusted ${ }^{\#}$ hazard ratios (HR) and $95 \%$ confidence intervals for the joint effects of maternal asthma and quartiles of nitrogen dioxide $\left(\mathrm{NO}_{2}\right)$ exposure over specific periods of pregnancy on childhood asthma risk

\begin{tabular}{|c|c|c|c|c|c|}
\hline Maternal asthma & $\mathrm{NO}_{2}$ quartiles & Subjects & Obstetric cases & HR $(95 \% \mathrm{CI})$ & RERI $(95 \%$ CI) \\
\hline 1st trimester & & & & & $0.08(-0.02-0.18)$ \\
\hline No & Q1 & 56939 & 7142 & Ref. & \\
\hline No & Q3 & 54914 & 8499 & $1.05(0.99-1.11)$ & \\
\hline No & Q4 & 55204 & 9613 & $1.10(1.02-1.18)$ & \\
\hline Yes & Q1 & 3951 & 767 & $1.59(1.48-1.71)$ & \\
\hline Yes & Q4 & 2821 & 765 & $1.77(1.67-1.97)$ & \\
\hline 2nd trimester & & & & & $0.18(0.08-0.28)$ \\
\hline No & Q1 & 56836 & 7156 & Ref. & \\
\hline No & Q2 & 55541 & 7593 & $1.02(0.98-1.06)$ & \\
\hline No & Q3 & 54512 & 8290 & $1.09(1.04-1.15)$ & \\
\hline No & Q4 & 54660 & 9504 & $1.16(1.08-1.26)$ & \\
\hline 3rd trimester & & & & & $0.07(-0.03-0.16)$ \\
\hline No & Q1 & 56139 & 7147 & Ref. & \\
\hline No & Q2 & 55097 & 7669 & $1.00(0.96-1.04)$ & \\
\hline No & Q3 & 54221 & 8207 & $1.02(0.96-1.07)$ & \\
\hline No & Q4 & 54247 & 9171 & $1.06(0.98-1.15)$ & \\
\hline Yes & Q1 & 3851 & 745 & $1.54(1.43-1.66)$ & \\
\hline Yes & Q2 & 3240 & 671 & $1.56(1.44-1.70)$ & \\
\hline Yes & Q3 & 2953 & 696 & $1.61(1.47-1.77)$ & \\
\hline Yes & Q4 & 2794 & 748 & $1.66(1.49-1.81)$ & \\
\hline Entire pregnancy & & & & & $0.06(-0.04-0.15)$ \\
\hline No & Q1 & 54748 & 6928 & Ref. & \\
\hline No & Q2 & 52430 & 6908 & $0.95(0.91-1.00)$ & \\
\hline No & Q3 & 52598 & 7964 & $1.03(0.97-1.09)$ & \\
\hline No & Q3 & 51107 & 7625 & $1.06(1.01-1.12)$ & \\
\hline No & Q4 & 50074 & 9102 & $1.12(1.04-1.21)$ & \\
\hline Yes & Q1 & 3731 & 727 & $1.55(1.43-1.67)$ & \\
\hline Yes & Q2 & 2977 & 562 & $1.60(1.48-1.72)$ & \\
\hline Yes & Q3 & 2523 & 668 & $1.68(1.53-1.83)$ & \\
\hline Yes & Q4 & 2427 & 717 & 1.75 (1.59-1.92) & \\
\hline
\end{tabular}

Data are presented as $n$, unless otherwise stated. $Q$ : quartile; RERI: relative excess risk of childhood asthma due to interaction between maternal asthma and $\mathrm{NO}_{2}$. ${ }^{\#}$ : models adjusted for maternal age at delivery, infant sex, parity, breastfeeding status at the time of discharge, maternal smoking during pregnancy, gestational age, birthweight, residential greenness exposure during pregnancy, dissemination area median family income, dissemination area proportion of population who are visible minority, dissemination area proportion of the adult female population aged 25-64 years who completed post-secondary education, the average pregnancy exposure to the selected pollutant, the first year of life exposure to the selected pollutant and cumulative exposure after birth to the selected pollutant.

We found evidence that impacts on childhood asthma diagnosed before 6 years of age increased in a synergistic manner when evaluating the joint effect of maternal asthma and high levels of exposure to $\mathrm{NO}_{2}$ during the second trimester of pregnancy. To our knowledge, no previous study has investigated this important issue. Prior literature has shown that inhalation of gaseous pollutants can induce pro-inflammatory processes in the lungs of pregnant females [40]. Inflammation is a characteristic feature of the pathophysiology of asthma [17]. It is therefore biologically plausible that inflammation from 
TABLE 6 Adjusted ${ }^{\#}$ hazard ratios (HR) and $95 \%$ confidence intervals for the joint effects of maternal asthma and quartiles of particulate matter with a $50 \%$ cut-off aerodynamic diameter $\leqslant 2.5 \mu \mathrm{m}$ (PM2.5) exposure over specific periods of pregnancy on childhood asthma risk

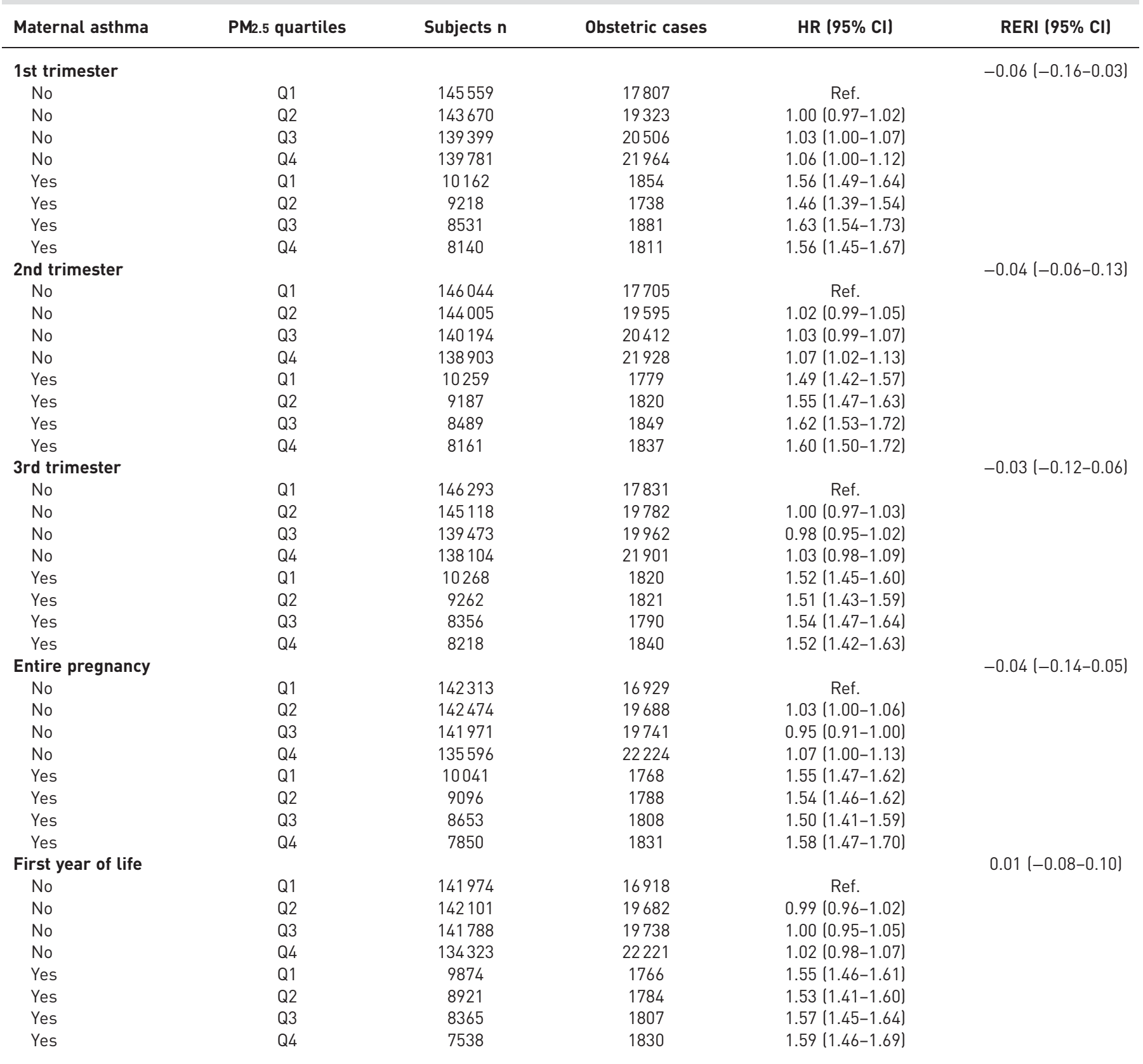

Data are presented as $n$, unless otherwise stated. $Q$ : quartile; RERI: relative excess risk of childhood asthma due to interaction between maternal asthma and PM2.5. \#: models adjusted for maternal age at delivery, infant sex, parity, breastfeeding status at the time of discharge, maternal smoking during pregnancy, gestational age, birthweight, residential greenness exposure during pregnancy, dissemination area median family income, dissemination area proportion of population who are visible minority, dissemination area proportion of the adult female population aged 25-64 years who completed post-secondary education, the average pregnancy exposure to the selected pollutant, the first year of life exposure to the selected pollutant and cumulative exposure after birth to the selected pollutant.

exposure to air pollution during pregnancy combined with inflammation due to maternal presence of asthma induces a synergistic effect on childhood asthma development. This could occur through an alteration of immune responses [41]. Additionally, our findings could reflect the fact that traffic pollution may potentiate airway inflammation in already sensitised children through an epigenetic pathway (i.e. those with a genetic susceptibility to develop asthma) [42]. These findings require further investigation. 
Our results confirm the previously reported finding that children weighing $<2500 \mathrm{~g}$ at birth were at higher risk of developing asthma during childhood following exposure to air pollution during the gestational period [11]. In addition, we found stronger impacts from exposure to air pollution on development of childhood asthma among children born preterm compared to those born full-term, and those born to mothers living in urban areas during pregnancy compared to rural areas. This finding is relevant as it shows greater susceptibility among those living in urban areas [43]. This may relate to the "hygiene hypothesis", where living in urban areas characterised by wealthy lifestyles and wealthy housing characteristics may increase risk of developing asthma. In addition, a protective effect of microbial exposures from rural environments has been reported [44]. We found higher effects for those born to mothers who smoked during pregnancy. Our findings for higher effects of prenatal exposure to PM2.5 on childhood asthma incidence among males is consistent with one previous study $[9,14]$.

Some limitations of our study need to be acknowledged. First, we could assign $\mathrm{NO}_{2}$ exposures only to participants that were within $25 \mathrm{~km}$ of a ground monitor in order to apply the temporal scaling described in the online supplementary material. This decreased the sample sizes for analysis when investigating $\mathrm{NO}_{2}$ exposures, but probably reduced the likelihood of misclassification bias in exposure [45]. Second, while we included a number of important confounding factors, we cannot rule out potential residual confounding. For example, we did not have individual-level information on ethnicity, income, education or maternal stress levels. However, adjustment for area-level SES factors may have partially accounted for confounding for some of these missing variables. We did not have information on maternal obesity in pregnancy or maternal gestational weight gain, both of which are important risk factors for childhood asthma development [46]. However, a sensitivity analysis among a subset of our cohort (i.e. $\sim 20 \%$ of our cohort) with information on maternal pre-pregnancy body mass index indicated that adjustment for this factor did not materially change the main effect estimates (results not shown). Another limitation is related to the fact that we identified cases of asthma based on health administrative databases, which may lead to some level of misclassification bias. For example, we did not have information on asthma phenotypes and asthma severity. We also did not have information on medications used to treat or control asthma during pregnancy. However, a recent Canadian study that used a similar physician-diagnosed asthma case ascertainment as our study showed that traffic-related air pollution was associated with asthma status that was maintained over a 10-year follow-up. Therefore, this provides support that we likely captured "true" asthma cases. In addition, children aged $<5$ years with symptoms of wheeze due to viral infections may be misdiagnosed as having asthma [23, 47]. However, we used a validated case definition in identifying asthma among both children and adults [30]. Finally, we could have underestimated asthma diagnosis in mothers since the data we used to identify maternal asthma went as far back as 1991, and therefore we would not identify those diagnosed with asthma during childhood. In addition, if these mothers had asthma during their early childhood and were well controlled afterwards without any documented healthcare use attributed to asthma, we would have missed them. Thus, we assume that most of the mothers with asthma captured through the health administrative database would be "prevalent" cases and/or may be those with relatively less well-controlled (or severe) asthma that would continue with encounters in the health care system during their adulthood [30].

Notable strengths of this study include the large sample size, availability of spatiotemporal air pollution exposure estimates available across a large geographical area and the attempt to capture residential mobility during pregnancy. The population-based approach is likely to have reduced risks of selection bias.

In this large population-based study, we found that exposure to ambient air pollution in pregnancy may increase the risk of asthma in young children. We observed enhanced effects of air pollution on the onset of childhood asthma diagnosed before 6 years of age among those born to mothers with a history of asthma, those born to mothers who smoked during pregnancy, in males and according to gestational age, birthweight and maternal place of residence. These findings highlight the need for further research to confirm relationships identified here and also the importance of developing public health and prenatal care strategies aimed at raising awareness and minimising exposure to ambient air pollution during pregnancy.

Acknowledgements: The authors thank Ann Sprague (Better Outcomes Registry and Network (BORN), Ottawa, ON, Canada) for facilitating access to the BORN database. No endorsement by the Institute for Clinical Evaluative Sciences (ICES) or the Ontario Ministry of Health and Long-Term Care is intended or should be inferred. The opinions, results and conclusions reported in this paper do not necessarily represent the views of BORN.

\section{References}

Asher I, Dagli E. Environmental influences on asthma and allergy. Chem Immunol Allergy 2004; 84: 36-101.

2 Subbarao P, Becker A, Brook JR, et al. Epidemiology of asthma: risk factors for development. Expert Rev Clin Immunol 2009; 5: 77-95. 
3 Khreis H, Kelly C, Tate J, et al. Exposure to traffic-related air pollution and risk of development of childhood asthma: a systematic review and meta-analysis. Environ Int 2016; 100: 1-31.

4 Gasana J, Dillikar D, Mendy A, et al. Motor vehicle air pollution and asthma in children: a meta-analysis. Environ Res 2012; 117: 36-45.

5 Morales E, Garcia-Esteban R, de la Cruz OA, et al. Intrauterine and early postnatal exposure to outdoor air pollution and lung function at preschool age. Thorax 2015; 70: 64-73.

6 Mortimer K, Neugebauer R, Lurmann F, et al. Air pollution and pulmonary function in asthmatic children: effects of prenatal and lifetime exposures. Epidemiology 2008; 19: 550-557.

7 Schultz ES, Litonjua AA, Melén E. Effects of long-term exposure to traffic-related air pollution on lung function in children. Curr Allergy Asthma Rep 2017; 17: 171.

8 Schultz ES, Hallberg J, Bellander T, et al. Early-life exposure to traffic-related air pollution and lung function in adolescence. Am J Respir Crit Care Med 2016; 193: 171-177.

9 Hsu HH, Chiu YH, Coull BA, et al. Prenatal particulate air pollution and asthma onset in urban children. identifying sensitive windows and sex differences. Am J Respir Crit Care Med 2015; 192: 1052-1059.

10 Clark NA, Demers PA, Karr CJ, et al. Effect of early life exposure to air pollution on development of childhood asthma. Environ Health Perspect 2010; 118: 284-290.

11 Sbihi H, Tamburic L, Koehoorn M, et al. Perinatal air pollution exposure and development of asthma from birth to age 10 years. Eur Respir J 2016; 47: 1062-1071.

12 Deng Q, Lu C, Norbäck D, et al. Early life exposure to ambient air pollution and childhood asthma in China. Environ Res 2015; 143: 83-92.

13 Deng Q, Lu C, Li Y, et al. Exposure to outdoor air pollution during trimesters of pregnancy and childhood asthma, allergic rhinitis, and eczema. Environ Res 2016; 150: 119-127.

14 Lee A, Leon Hsu HH, Mathilda Chiu YH, et al. Prenatal fine particulate exposure and early childhood asthma: effect of maternal stress and fetal sex. J Allergy Clin Immunol 2017; in press [https://doi.org/10.1016/j.jaci.2017.07.017].

15 Liu W, Huang C, Hu Y, et al. Associations of gestational and early life exposures to ambient air pollution with childhood respiratory diseases in Shanghai, China: a retrospective cohort study. Environ Int 2016; 92-93: 284-293.

16 Hehua Z, Qing C, Shanyan G, et al. The impact of prenatal exposure to air pollution on childhood wheezing and asthma: a systematic review. Environ Res 2017; 159: 519-530.

17 Litonjua AA, Carey VJ, Burge HA, et al. Parental history and the risk for childhood asthma. Does mother confer more risk than father? Am J Respir Crit Care Med 1998; 158: 176-181.

18 Castro-Rodriguez JA, Forno E, Rodriguez-Martinez CE, et al. Risk and protective factors for childhood asthma: what is the evidence? J Allergy Clin Immunol Pract 2016; 4: 1111-1122.

19 Lim RH, Kobzik L, Dahl M. Risk for asthma in offspring of asthmatic mothers versus fathers: a meta-analysis PLoS One 2010; 5: e10134.

20 Murphy VE. Managing asthma in pregnancy. Breathe 2015; 11: 258-267.

21 Rancière F, Bougas N, Viola M, et al. Early exposure to traffic-related air pollution, respiratory symptoms at 4 years of age, and potential effect modification by parental allergy, stressful family events, and sex: a prospective follow-up study of the PARIS birth cohort. Environ Health Perspect 2017; 125: 737-745.

22 Dunn S, Bottomley J, Ali A, et al. 2008 Niday Perinatal Database quality audit: report of a quality assurance project. Chronic Dis Inj Can 2011; 32: 32-42.

23 To T, Dell S, Dick PT, et al. Case verification of children with asthma in Ontario. Pediatr Allergy Immunol 2006; 17: 69-76.

24 Lavigne E, Yasseen AS 3rd, Stieb DM, et al. Ambient air pollution and adverse birth outcomes: differences by maternal comorbidities. Environ Res 2016; 148: 457-466.

25 Lavigne E, Bélair MA, Do MT, et al. Maternal exposure to ambient air pollution and risk of early childhood cancers: a population-based study in Ontario, Canada. Environ Int 2017; 100: 139-147.

26 Brunsdon C, Fotheringham AS, Charlton ME. Geographically weighted regression: a method for exploring spatial nonstationarity. Geogr Anal 1996; 28: 281-298.

27 van Donkelaar A, Martin RV, Spurr RJ, et al. High-resolution satellite-derived PM2.5 from optimal estimation and geographically weighted regression over North America. Environ Sci Technol 2015; 49: 10482-10491.

28 Hystad P, Setton E, Cervantes A, et al. Creating national air pollution models for population exposure assessment in Canada. Environ Health Perspect 2011; 119: 1123-1129.

29 Hystad P, Villeneuve PJ, Goldberg MS, et al. Exposure to traffic-related air pollution and the risk of developing breast cancer among women in eight Canadian provinces: a case-control study. Environ Int 2015; 74: 240-248.

30 Gershon AS, Wang C, Guan J, et al. Identifying patients with physician-diagnosed asthma in health administrative databases. Can Respir J 2009; 16: 183-188.

31 Rhew IC, Vander Stoep A, Kearney A, et al. Validation of the normalized difference vegetation index as a measure of neighborhood greenness. Ann Epidemiol 2011; 21: 946-952.

32 Chen H, Burnett RT, Copes R, et al. Ambient fine particulate matter and mortality among survivors of myocardial infarction: population-based cohort study. Environ Health Perspect 2016; 124: 1421-1428.

33 Nasari MM, Szyszkowicz M, Chen H, et al. A class of non-linear exposure-response models suitable for health impact assessment applicable to large cohort studies of ambient air pollution. Air Qual Atmos Health 2016; 9: 961-972.

34 Knol MJ, VanderWeele TJ. Recommendations for presenting analyses of effect modification and interaction. Int J Epidemiol 2012; 41: 514-520.

35 Hosmer DW, Lemeshow S. Confidence interval estimation of interaction. Epidemiology 1992; 3: 452-456.

36 Burri PH. Fetal and postnatal development of the lung. Annu Rev Physiol 1984; 46: 617-628.

37 Kato A, Favoreto S Jr, Avila PC, et al. TLR3- and Th2 cytokine-dependent production of thymic stromal lymphopoietin in human airway epithelial cells. J Immunol 2007; 179: 1080-1087.

38 Moffatt MF, Gut IG, Demenais F, et al. A large-scale, consortium-based genomewide association study of asthma. N Engl J Med 2010; 363: 1211-1221.

39 Nadeau K, McDonald-Hyman C, Noth EM, et al. Ambient air pollution impairs regulatory T-cell function in asthma. J Allergy Clin Immunol 2010; 126: 845-852. 
40 Vadillo-Ortega F, Osornio-Vargas A, Buxton MA, et al. Air pollution, inflammation and preterm birth: a potential mechanistic link. Med Hypotheses 2014; 82: 219-224.

41 Wright RJ, Visness CM, Calatroni A, et al. Prenatal maternal stress and cord blood innate and adaptive cytokine responses in an inner-city cohort. Am J Respir Crit Care Med 2010; 182: 25-33.

42 DeVries A, Wlasiuk G, Miller SJ, et al. Epigenome-wide analysis links SMAD3 methylation at birth to asthma in children of asthmatic mothers. J Allergy Clin Immunol 2017; 140: 534-542.

43 Asher MI. Urbanisation, asthma and allergies. Thorax 2011; 66: 1025-1026.

44 Wong GW, Chow CM. Childhood asthma epidemiology: insights from comparative studies of rural and urban populations. Pediatr Pulmonol 2008; 43: 107-116.

45 Bechle MJ, Millet DB, Marshall JD. National spatiotemporal exposure surface for $\mathrm{NO}_{2}$ : monthly scaling of a satellite-derived land-use regression, 2000-2010. Environ Sci Technol 2015; 49: 12297-12305.

46 Forno E, Young OM, Kumar R, et al. Maternal obesity in pregnancy, gestational weight gain, and risk of childhood asthma. Pediatrics 2014; 134: e535-e546.

47 Tétreault LF, Doucet M, Gamache P, et al. Childhood exposure to ambient air pollutants and the onset of asthma: an administrative cohort study in Québec. Environ Health Perspect 2016; 124: 1276-1282. 